\title{
Inline Oil Performance Monitor LUBRICON for Engines and Machines
}

\author{
Dr.-Ing. Bode, Berthold \\ flucon fluid control $\mathrm{GmbH}$ \\ Burgstätter Str. 6 \\ D-38678 Clausthal-Zellerfeld
}

\section{Introduction}

The optimization of the intervals in which lubricating liquids have to be exchanged is of growing importance for the continued technical development of automobiles, utility vehicles, gear construction and energy producing plants.

The unit-specific optimization of the intervals in which the lubricating liquid is exchanged contributes significantly to a decrease of pollution while it increases operational stability and thus reduces the cost of operation at the same time, as it means a controlled prolongation of the time in which the lubricant can be used, and therefore an increase in the time in which the machinery is available.

The necessary permanent control of the quality of the lubricating oil is now made possible by LUBRICON, which is the result of long years of research.

\section{Oil Performance and Characteristic Values of Lubricants}

The aging process of lubricants is largely determined by the appearance of contamination in the lubricant, thermal oxidization, chemical conversion, and mechanical destruction of additives of high molecular weight. Especially contamination depends on the specific application; typical contaminations are caused by particles (metals and siloxanes), hydrogen sulphide (acidic), carbon dioxide and water.

Aging of liquid lubricants is essentially characterized by such quantifiable phenomena as the change of flowability, an increase of insoluble impurities (including metallic particles), and a change in the capacity for neutralization (Total Base Number „TBN“ and / or Total Acid Number „TAN“, respectively).

As demonstrated by our research the change of viscosity in the lubricant is of major importance; while the obtaining of such operational data as the change of the relative permittivity and the specific electric conductivity provides conclusions concerning the chemical changes in the lubricant.

The combination of these measurement data by use of the LUBRICON system consequently leads to information about the remaining performance of the lubricant.

\section{Fundamentals of the Oil Performance Monitor LUBRICON}

The measuring device LUBRICON consists of two components: The screw-inserted sensor and a small compartment with the electronic unit as shown in Figure 1.

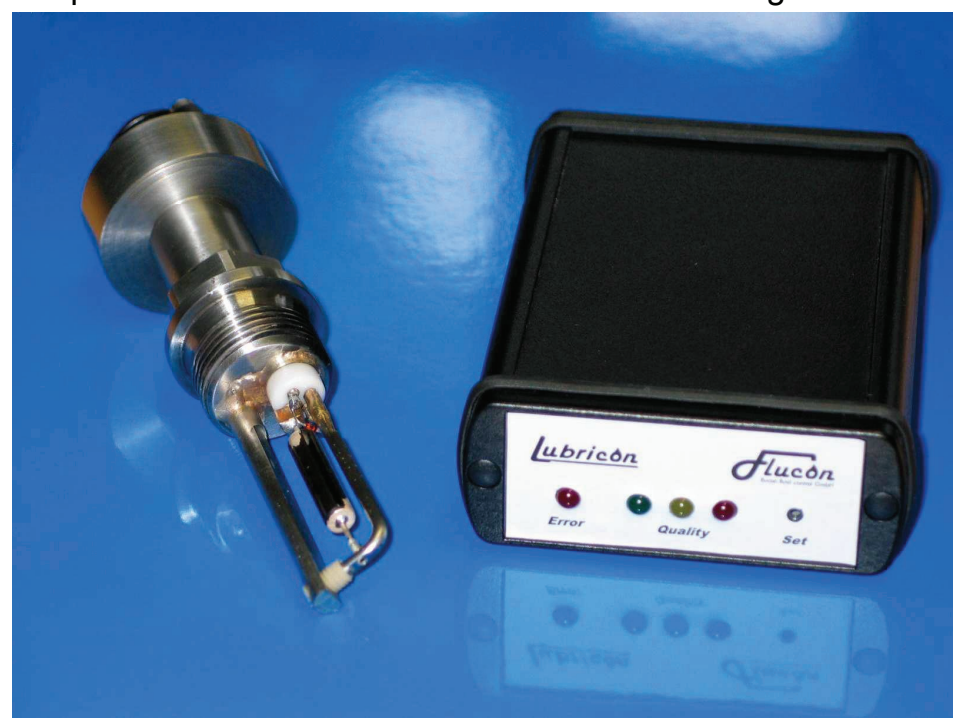

Figure 1: Oil Performance Monitor LUBRICON (Sensor \& Electronic Unit) 
Besides the actual temperature the following three characertistic values of the oil are continuously measured within a response time of approximately 20 seconds:

\section{a. Viscosity}

During the time in which the lubricant is used there are essentially three different processes that combine their effects and determine the aging of the lubricant:

- The mechanical destruction of high-polymer VI-improvers causes an immediate decrease of the viscosity and a deterioration of the viscosity-temperature (VT)-relation..

- At the same time there is a process of thermal oxidization which - depending on the temperature range that the liquid is subjected to - may lead to a continuous rising of the flowability; this effect is supported by the increasing exhaustion of the specific oil additives.

- While the lubricant is in use there is always an appearance of particles with their quality and quantity, depending on the specific application. While they may be external impurities, or the result of friction or of combustion, the consequence is always an increase of viscosity.

\section{b. Relative Dielectric Constant / Permittivity}

Free load carriers in the liquid are a prerequisite for the flow of electric power. But even if free load carriers are not available the liquid will react under the influence of an electric field; an effect observed by FARADAY on the basis of a capacitor filled with liquid and described in terms of the so-called dielectric properties of a medium. The permittivity of air is 1.0, the typical value for oil and gasoline is 2.3 while the value for water is 80.0 . Because of the large difference between oil and water the permittivity value is an ideal indicator of the water content in the used oil. With the LUBRICON-sensor the electrodes are positioned in such a way as to furnish a capacitor whose electric field is influenced by the characteristics of the liquid, a fixed frequency providing a permanent electric potential.

\section{c. Specific Electric Conductivity}

If there are only free charge carriers in the liquid the effect of an electric potential upon it is a current conduction equivalent to the existing potential difference. The fluid behaves as an ohmic resistor, the output yielded being a purely real part of energy.

However all lubricating oils have a comparatively low degree of electric conductivity, so that they are generally considered as belonging to the group of non-conductors.

As the molecular structure of the fresh lubricant in combustion engines changes due to wear and according to duration and mode of application, changes of electric conductivity also occur in the liquid; these are of a very limited extent, however, requiring for their detection a highly sensitive and precise measuring device. The electrical conductivity of a basically non-conductive liquid is necessarily very low, - in the MOhm-range of the equivalent circuit diagram of the impedance of the liquid. The increasing level of the specific electric conductivity is caused by the accumulation of acids in the lube oil which is equivalent to an increase of the liquid's Total Acid Number „TAN“.

The most important component of the LUBRICON system is the cylinder-shaped piezoelectric torsional quartz sensor which offers a lot of advantages for the inline application:

- one single sensor only for the detection of the three main characteristic values of oil

- independent of type of flow (still/laminar/turbulent)

- small dimensions ( $\mathrm{d}=6 \mathrm{~mm}, \mathrm{l}=25 \mathrm{~mm})$

- no wear as there are no moveable parts

- no maintenance and service costs

- high degree of installation flexibility

- automatic measurement

- highly resistant to contamination and sedimentation. 
The following Figure 2 shows the outline of the LUBRICON sensor with 3/4"-adapter.

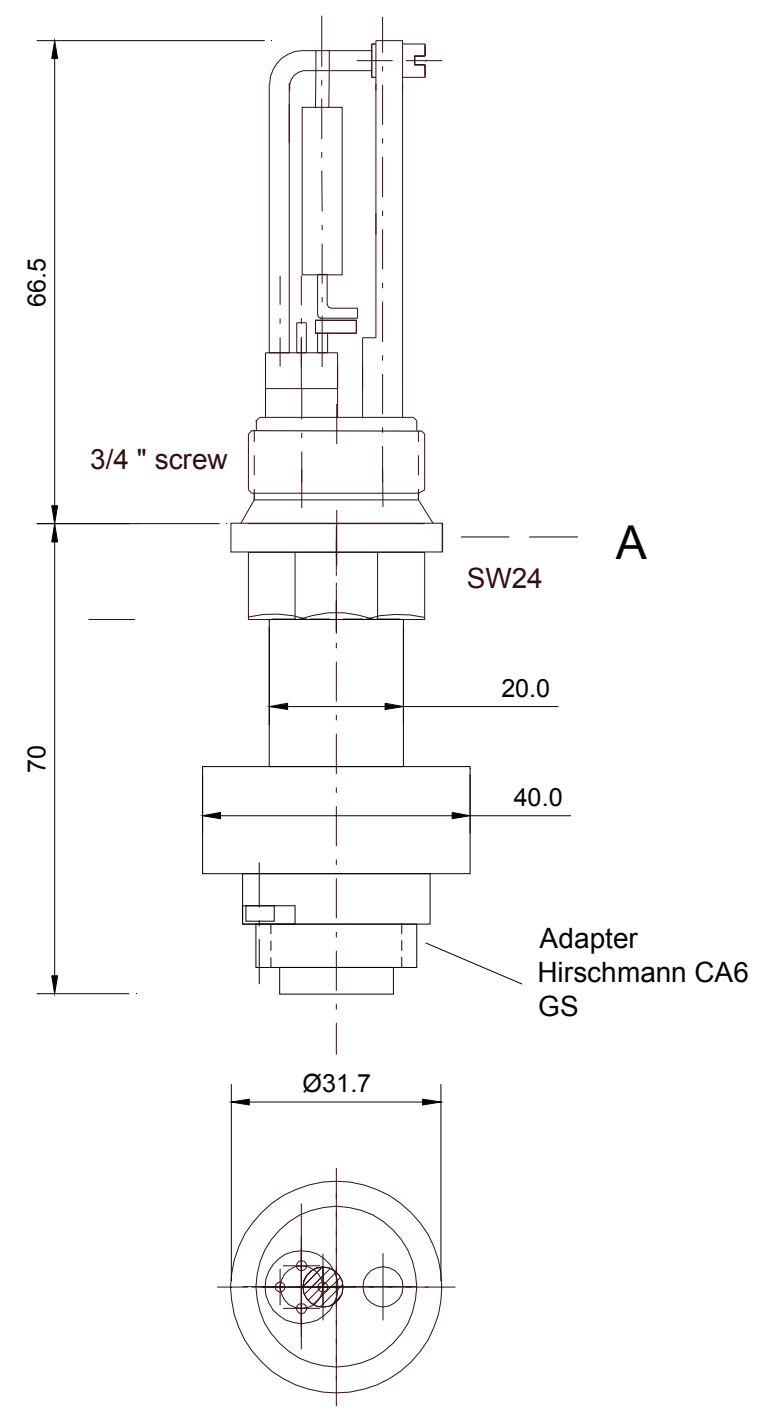

Figure 2: LUBRICON Sensor (Drawing)

\section{System Application and Functions}

The measuring system LUBRICON serves two fundamental purposes: a continuous measuring of all the oil parameters with data output obtained at the digital USB interface (with additional analog interface output $4 \ldots 20 \mathrm{~mA} / 1 \ldots 5 \mathrm{~V}$ optionally avalaible), and the monitoring of oil quality according to the requirements determined by the user, who can enter his requirements into the system as part of the programming.

The system provides the continuous measuring of the following four types of operational data:

- oil temperature

- viscosity

- relative dielectric constant (permittivity)

- specific electric conductivity.

In addition to the continuous measuring of all relevant oil data LUBRICON can also provide monitoring of oil quality, which takes place on the basis of the specific condition of the fresh oil.

Three different levels of oil quality can be indicated by the system:

- good / very good quality (LED green)

- medium quality (LED yellow)

- bad quality (LED red). 
As far as the definition of oil quality is concerned, fresh oil is assigned a value of $100 \%$; when oil quality reaches a value of $\leq 0 \%$ the oil must by all means be exchanged.

After fresh-oil calibration has taken place the quality of the oil will be indicated by means of the three LEDs; for this purpose the following limit values of maximum oil strain have been defined:

- limit value „Viscosity [\%]“

- limit value "Relative Dielectric Constant [\%]“

- limit value „Specific Electric Conductivity [\%]“.

These limit values represent a deviation in percent on the basis of the fresh-oil under consideration; they are absolute values, i.e. they can denote values that are either higher or lower than the data obtained from the fresh oil.

Finally the three characteristic values of the oil , - each based on a comparison to the respective fresh-oil data -, will be combined by means of superposition to indicate oil quality.

Nevertheless it is necessary that every user establishes the correspondence between the signal and the level of oil quality himself, as only the particulars of application can determine, which oil quality is unacceptable. In other words: The user is responsible to tell the system at what point it should signal "red = oil quality bad“".

The general procedure is as follows:

$\rightarrow$ After fresh oil has been fed into the system the command "fresh-oil calibration" activates the LUBRICON system, which then records the changes of the four different measuring values and determines the oil quality by means of the preset specified limit values.

$\rightarrow$ Right before the next change of oil this data can be read and then serve as the basis for individual reseting. The definition of the oil quality should be undertaken on the background of the chemical analysis of the oil on the one hand and the given oil specifications of the machine manufacturer on the other.

$\rightarrow$ In consequence, the LUBRICON system will indicate the three different levels of oil quality for the individual application; the users are able to create their own presetting according to their needs and requirements, - the optimal monitoring of oil against premature aging can be achieved.

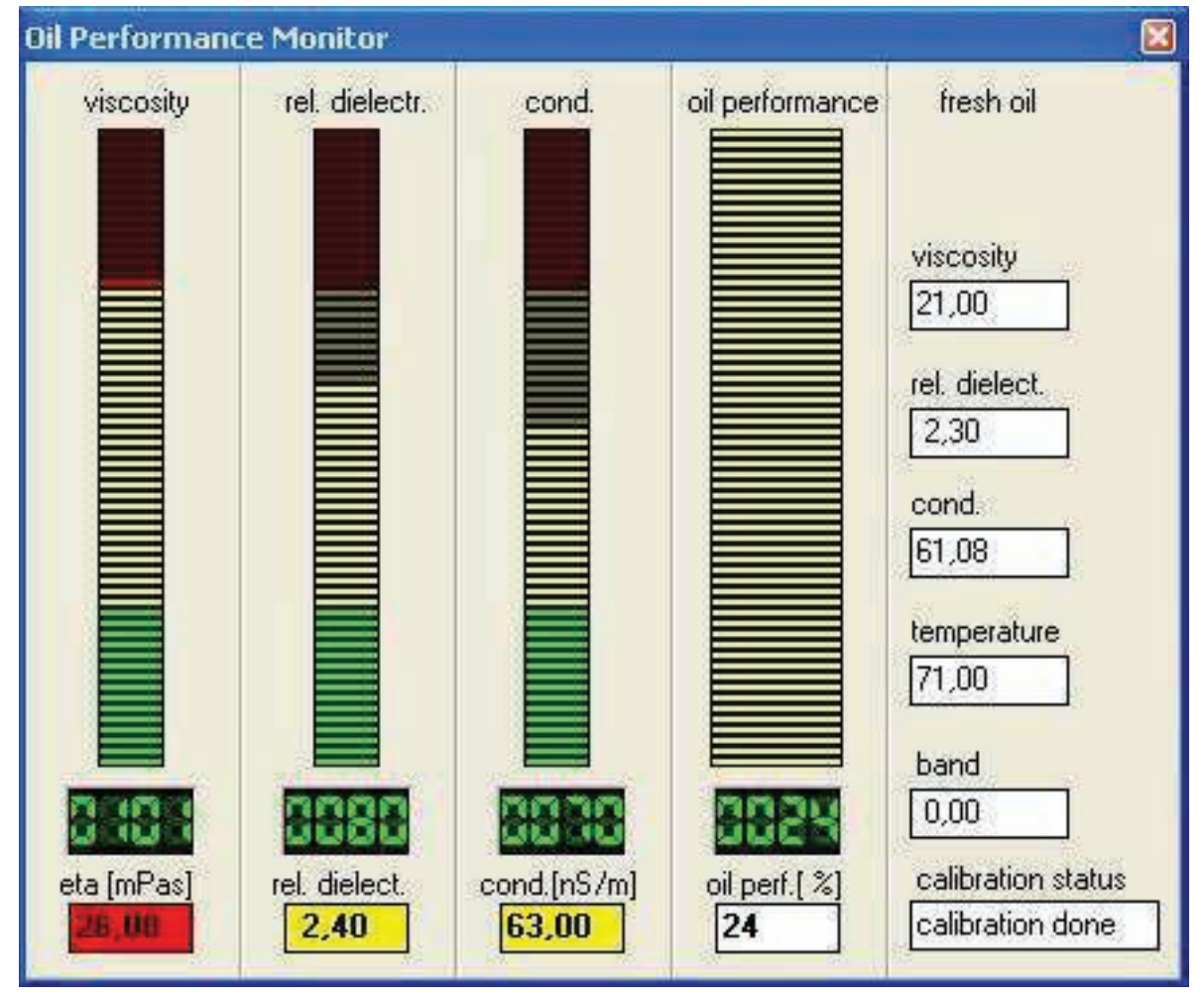

Figure 3: Oil Performance by Use of the LUBRICON Monitor 


\section{LUBRICON System in Practical Application (Typical Measuring Results)}

The LUBRICON system has been successfully applied in the monitoring of diesel-operated block heat and power plants, diesel and bio-fuel operated truck engines and gas resp. biogas plants.

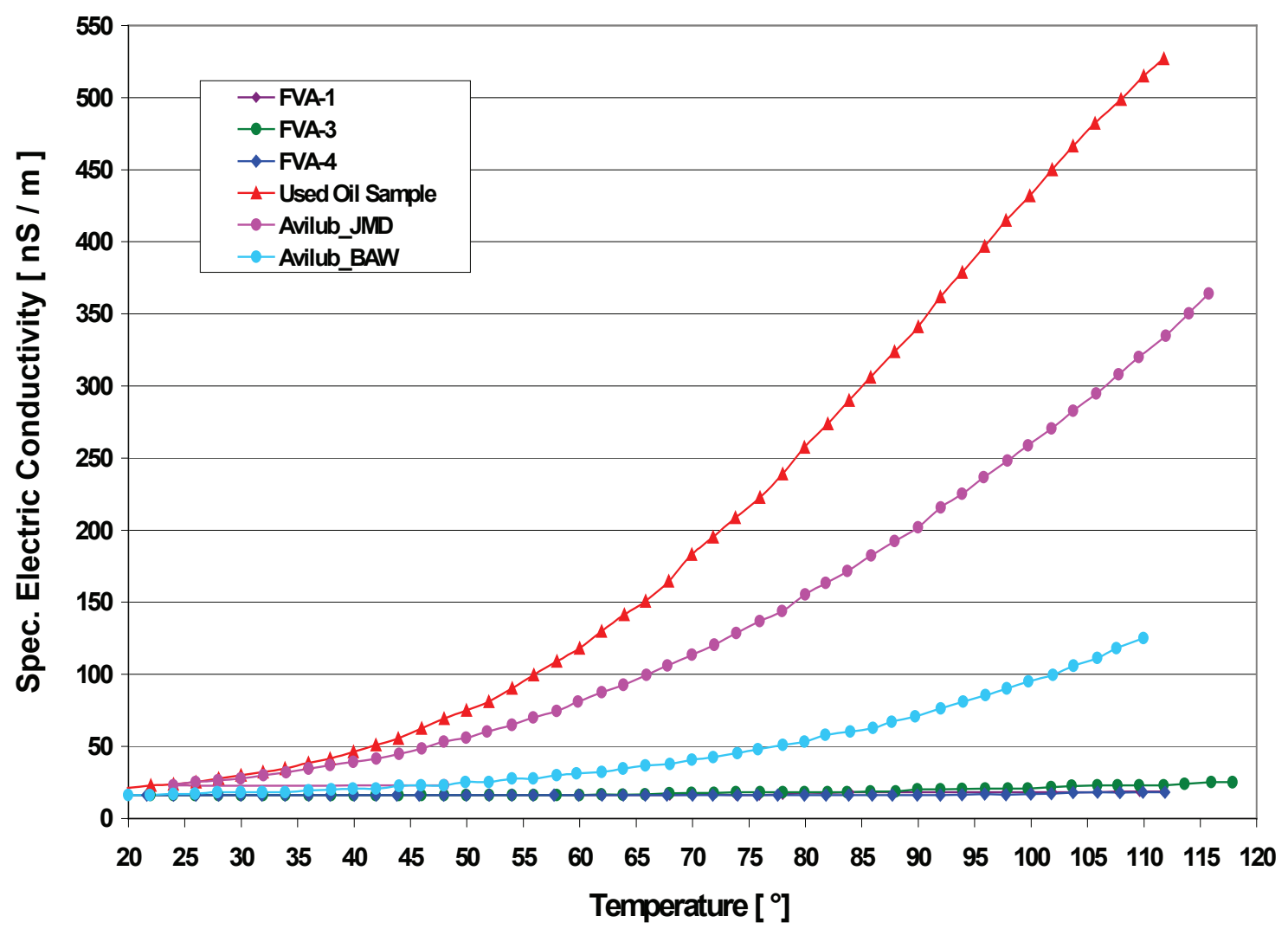

Figure 4: Specific Electric Conductivity of Fresh and Used Oils

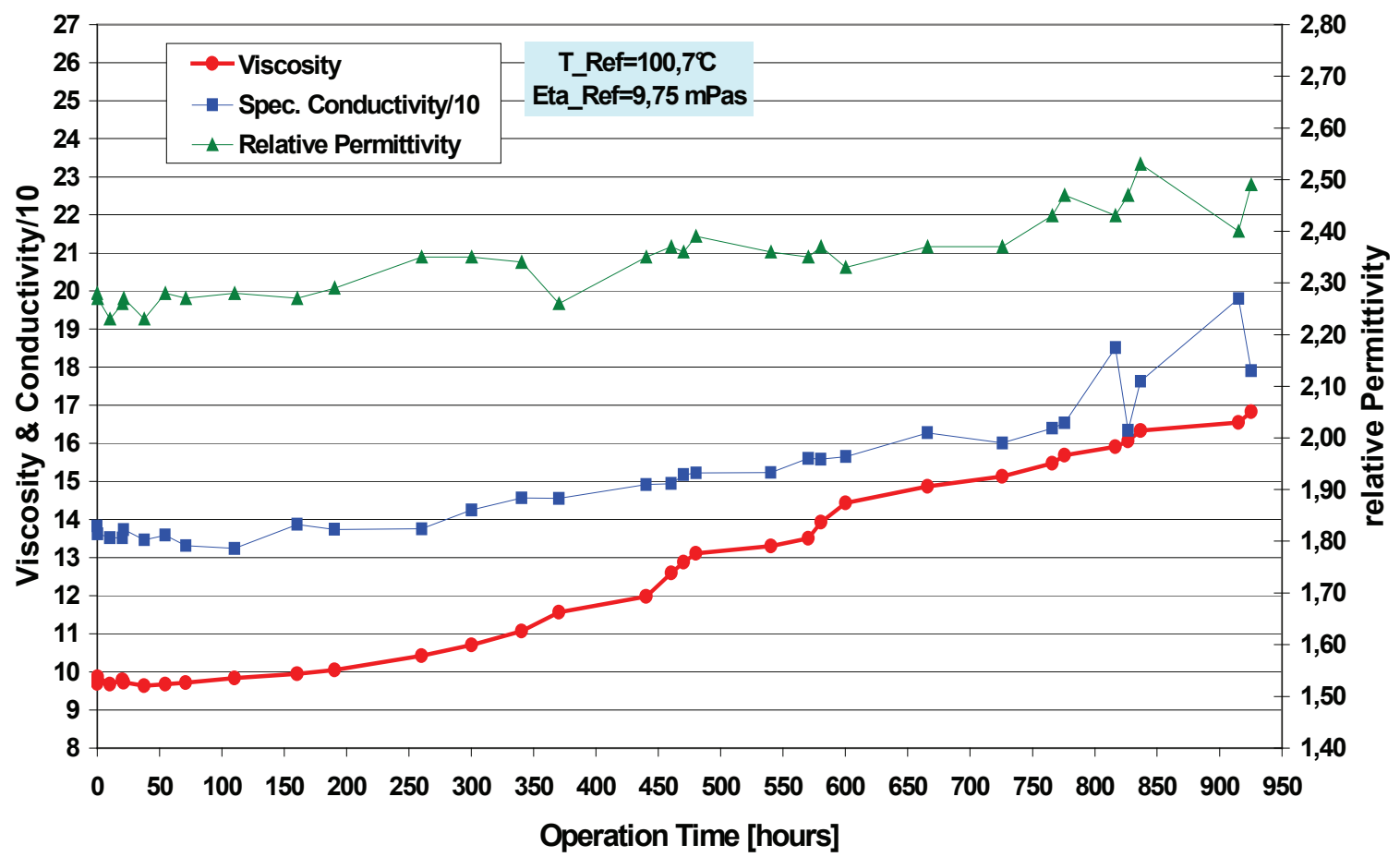

Figure 5: Datalogger Cycle of LUBRICON-Inline Measurement (Biogas Plant "Binder") 